Voix et Images

voixetimages

\title{
La part prophétique dans les premiers romans de Gabrielle Roy
}

\section{Richard M. Chadbourne}

Volume 14, numéro 3 (42), printemps 1989

Gabrielle Roy

URI : https://id.erudit.org/iderudit/200793ar

DOI : https://doi.org/10.7202/200793ar

Aller au sommaire du numéro

Éditeur(s)

Université du Québec à Montréal

ISSN

0318-9201 (imprimé)

1705-933X (numérique)

Découvrir la revue

Citer cet article

Chadbourne, R. M. (1989). La part prophétique dans les premiers romans de Gabrielle Roy. Voix et Images, 14(3), 399-407. https://doi.org/10.7202/200793ar d'utilisation que vous pouvez consulter en ligne.

https://apropos.erudit.org/fr/usagers/politique-dutilisation/ 


\title{
La part prophétique dans les premiers romans de Gabrielle Roy
}

\author{
par Richard M. Chadbourne, Université de Calgary
}

Vos fils et vos filles prophétiseront.

Joël 2:29, repris par saint Pierre dans Actes 2: 17-18.

Les êtres de génie sont contemporains de l'avenir plus que de leur propre temps.

Jean Guitton, Génie de Pascal, p. 11.

C'est un des privilèges du créateur littéraire, surtout du romancier, que d'imaginer comme existantes des situations que seul l'avenir va réaliser, mais dont les germes sont cachés dans le présent. De ce pouvoir visionnaire ou prophétique, Balzac est l'un des plus célèbres exemples. Oscar Wilde, dans un de ces épigrammes qu'il affectionnait, en forme de boutade masquant une vérité profonde, a écrit: La littérature anticipe toujours la vie; loin de la copier, elle la façonne à son but. Le dix-neuvième siècle, tel que nous le connaissons, est en grande partie une invention de Balzac. ${ }^{1}$ Loin de moi de vouloir réclamer pour Gabrielle Roy l'invention du Québec contemporain ou des intuitions de l'avenir ou du monde invisible du même ordre génial que l'on trouve dans l'œuvre du Balzac «visionnaire» ou dans celle du Victor Hugo dont les yeux plongeaient plus loin que le monde réel («Extase»). Mais qu'il y ait chez Gabrielle Roy un don réel de pénétration dans l'avenir a déjà été signalé par plusieurs critiques, notamment par Georges-André Vachon, Ben-Zion Shek et Paul Socken.

Vachon, dans un article de 1966, conclut que toute l'auvre [de Gabrielle Roy] annonce, avec une avance d'une quinzaine d'années, les transformations de l'espace politique et social que le Québec devait subir, tous les conflits de l'idéologie nationaliste, sans cesse contestée par la réalité historique ${ }^{2}$. Pour Socken, le concept régien du pays de l'amour (ne serait-ce qu'un autre nom pour «le Royaume de Dieu»?) représente la somme de valeurs idéalisées qui, tout en se rattachant en partie à un passé regretté (a less complicated rural past),

1 Oscar Wilde, «The Decay of Lying», dans Intentions and Other Writings, Garden City, New York, Doubleday, [s.d.], p. 31. La traduction est de moi.

2 Georges-André Vachon, «L'espace politique et social dans le roman québécois», Recherches sociographiques, vol. VII, no 3, septembre-décembre 1966, p. 261. 
sont fondées en même temps sur a visionary sense of what the world should strive to attain if it is to fully realize itself ${ }^{3}$.

Employant l'adjectif «prophétique» dans un autre sens, François Hébert, au cours d'un compte rendu de plusieurs romans québécois de l'année 1973, parle de la part prophétique dans ces ouvrages - parmi eux, Cet été qui chantait où l'auteure cherche d restaurer, par son ouvre, d' une manière ou d' une autre, le sens du sacrét.

Mais ce qui m'intéresse avant tout ici, c'est «l'avant-gardisme» de Gabrielle Roy concernant l'Église catholique, son pouvoir d'être «contemporaine de l'avenir» en pressentant et en imaginant des transformations que le concile Vatican II devait promulguer. Dans certains de ses livres, il faut le dire, elle voit si loin qu'elle laisse derrière elle les réformateurs. Que l'on songe à ses méditations sur l'artiste devant Dieu (la Montagne secrète) ou, inspirés en partie par Teilhard de Chardin, à ses propos sur l'harmonie que l'humanité doit urgemment chercher à rétablir avec la nature (la Petite Poule d'eau, Cet été qui chantait, surtout «La messe aux hirondelles»). Voilà deux questions voisines de la théologie: une théologie de l'art, une théologie de l'écologie - questions qui attendent toujours d'être mises à l'ordre du jour d'un concile futur... Mais, écartant ces questions pour des raisons pratiques, je me limiterai ici, poursuivant quelques remarques suggestives de Ben-Zion Shek, à deux des réformes conciliaires qui sont en voie d'accomplissement et qui avaient été anticipées par notre auteure: l'œcuménisme et l'apostolat du laïc.

Dans un article de 1971, Shek remarque:

Le thème religieux est très important dans Alexandre Chenevert et il nous semble que la critique de la religion traditionnelle dans ce roman est elle aussi très significative et annonciatrice de la mise en question générale qui a frappé l'Église catholique au Québec à partir surtout des années soixante. 5

Dans son Social Realism in the French-Canadian Novel, il retourne à cet aspect du roman en soulignant la portée annonciatrice non pas seulement du theme of cultural alienation, mais aussi de Chenevert's forceful critique of the Catholic Church [...] clearly a harbinger of the aggiornamento that came partially to that important Quebec institution in the 1960s ${ }^{6}$.

$J$ 'interroge le monde, dit Gabrielle Roy dans une interview citée par Marc Gagné, $j$ 'interroge l'univers, $j$ 'interroge aussi Dieu. Je prends souvent Dieu a

3 Paul Socken, «Le pays de l'amour in the works of Gabrielle Roy», Revue de l'Université d'Ottawa, vol. XLVI, n` 3, juillet-septembre 1976, p. 323.

4 François Hébert, «Le roman: de quelques avatars de Dieu», Etudes françaises, vol. IX, no 4, novembre 1973, p. 346.

5 Ben-Zion Shek, «L'espace et la description symbolique dans les romans montréalais de Gabrielle Roy», Liberté, no 73 (vol XIII, no 1), 1971, p. 93.

6 Ben-Zion Shek, Social Realism in the French-Canadian Novel, Toronto, Harvest House, 1977, p. 201-202. 
partie. $^{7}$ Interroger Dieu, Le prendre à partie: une très ancienne tradition, celle des prophètes bibliques. L'interroger surtout pour mieux savoir ce qu'Il veut pour Son peuple, pour mieux guider Son peuple - intention qui n'est pas non plus étrangère à l'œuvre de Gabrielle Roy.

La partie prophétique de cette œuvre me semble surtout frappante dans les trois premiers romans. Le troisième, Alexandre Chenevert, fut publié en 1954, huit ans avant l'ouverture de Vatican II (1962).

Bonheur d'occasion ne nous retiendra pas longtemps, car la vision du catholicisme qu'il contient est surtout négative - ou si l'on veut, prémonitoire. L'absence de vraie foi pèse sur le récit comme l'absence de Dieu dans certains romans de Mauriac. S'est substituée à elle la religiosité. Absents, en particulier, 'les ministres de cette foi, dans un véritable «tiers-monde» microcosmique, le monde des pauvres de Saint-Henri, avant que l'Église n'ait affirmé son «option pour les pauvres». La foi ne survit que très faiblement dans ce ghetto préconciliaire. Un christianisme déformé, mutilé, très courant au Québec d'avant le concile, légèrement caricaturé, mais somme toute assez bien représenté par la vieille madame Laplante qui s'était muée en une négation obstinée de tout espoir, attendant quand même, grâce à ses bonnes actions et ses indulgences, de monter au ciel, après avoir enduré son purgatoire sur terre. Quant au bonheur, elle n'y avait jamais cru (BO, p. 197). Une "chrétienne» qui ne croit pas au bonheur! Des «chrétiens», leur Église à leur tête, qui acceptent avec fatalisme le salut par la guerre (p. 378). Combien l'auteure nous fait sentir, dans le «soustexte» de ce roman, par la force de sa vision négative, l'urgence d'un retour à l'Évangile!

Aucun «dolorisme», aucun fatalisme dans la Petite Poule d'eau. Selon la belle formule du porte-parole de l'auteure, le père Joseph-Marie, la douleur du monde restait pour lui intacte, toujours indéchiffrable; mais de même la joie et l'amour (p. 243). La joie et l'amour, à vrai dire, dominent. Ils proviennent - c'est un fait sur lequel on n'a peut-être pas assez insisté - autant de l'expérience de l'Évangile vécue par les personnages que de leur communion avec la nature.

L'auteure a décrit son deuxième roman comme un peu une utopie ${ }^{8}$. Il y a utopies et utopies. Dans le cas de la Petite Poule d'eau (un lieu, soit dit en passant, car c'est très révélateur, qui existe sur la carte), utopie signifie non pas une conception chimérique, impossiblement idéalisée de la vie, mais la mise en valeur dans le présent de certaines possibilités de ce que pourrait être l'avenir. Ici comme ailleurs dans l'œuvre régienne, sont maintenues en tension les «deux exigences» analysées par François Ricard, la quête de l'idéal et la reconnaissance du réel9. En interprétant ce roman, on a peut-être trop insisté sur son

7 Marc Gagné, Visages de Gabrielle Roy, Montréal, Beauchemin, 1973, p. 247.

8 Cité par François Ricard dans Gabrielle Roy, Montréal, Fides, 1975, p. 65.

9 François Ricard, «Gabrielle Roy: le cercle enfin uni des hommes», Liberté, no 103 (vol. XVIII, no 1), janvier-février 1976, p. 62. 
indubitable charme idyllique et nostalgique - la joie qu'éprouve l'auteure à retrouver son pays manitobain après Bonheur d'occasion 10; on n'a pas suffisamment mis en relief les prévisions lucides de l'avenir. Il y a sans aucun doute ce que Vachon appelle le retour [...] d une certaine essence, supposée française, catholique et rurale, de l'être québécois ${ }^{11}$. Mais cet aspect du roman me semble subordonné à une vision de l'avenir. Variation sur le mythe du Paradis, incontestablement, que ce pays [...] intact, comme d peine sorti des songes du créateur - donc tourné vers le passé; mais c'est également le lieu où les hommes pourraient peut-être, s'ils le voulaient, recommencer à neuf (FLT, p. 196-197). Selon le genre d'utopie pratiqué par Gabrielle Roy, le «nulle part» actuel contient le «quelque part» de demain: il s'agit en l'occurrence de rien moins que du présent et de l'avenir de l'Église catholique.

Se dégagent de ce roman deux motifs prophétiques principaux intimement liés l'un à l'autre: le rôle approfondi du laïc - ici, de la femme - dans l'apostolat et le rôle plus humanisé du prêtre, surtout dans le contexte d'un œcuménisme grandissant. L'enthousiasme avec lequel l'auteure développe ces motifs est d'autant plus vif qu'elle semble se libérer ici, par l'imagination, du souvenir d'une Église qui tyrannisait les femmes sous prétexte de les mieux respecter et dont les prêtres formaient une caste à part.

Déjà chez Luzina Tousignant abondent cet amour universel et cette ouverture d'esprit sans lesquels l'œucuménisme promu par les pères du concile serait impossible. Le voyage l'avait instruite d' une façon inattendue; il lui avait enseigné que la nature est partout excellente en la remplissant d'un sentiment de bienveillance [...] qui englobait d peu près tous les humains, n'excluant même pas les Juifs (PPE, p. 32). Elle rencontre chez le père JosephMarie, le capucin de Toutes-Aides, un frère chrétien complètement à son diapason. Charismatique avant la lettre, il apprend de nombreuses langues, toujours avide de converser avec les gens qu'il rencontrait, qu'ils fussent ses paroissiens ou non (p. 170). (L'ouverture d'esprit de ces deux personnages nous fait penser un peu au pape Jean XXIII dont les voyages avant son élection l'avaient aussi instruit d' une façon inattendue et, dans son cas à lui, avaient nourri son esprit du projet du concile.)

Sans jamais paraître nier son vou de célibat, le capucin n'est pas sans connaître la nostalgie de la paternité (p. 240). Confessant Luzina, il lui fait écarter toute idée de péché lorsqu'elle lui avoue vouloir espacer un peu plus les naissances (p. 241). Le tour de son mari venu, le confesseur se trouve interdit devant la pensée que l'Église a canonisé tant de célibataires et si peu de pères de famille, et se demande pourquoi après avoir inspiré l'amour indispensable à ses desseins, Dieu n'en paraissait pas moins préférer ceux qui s'en abstenaient (p. 245).

10 Voir par exemple Gilles Marcotte, «Gabrielle Roy retoume à ses origines», le Devoir, 25 novembre 1950, p. 18.

11 Georges-André Vachon, loc. cit., p. 265. 
Le point culminant du roman est la messe annuelle dite par le capucin dans la maison des Tousignant, comme on célébrait l'Eucharistie parmi les premières générations de chrétiens. Dans la préparation de cette messe, Luzina joue ùn rôle actif, accueillant le capucin comme un membre de sa famille, envoyant un émissaire pour avertir ses voisins, arrangeant convenablement une des salles:

\begin{abstract}
Un élan d'affection toute maternelle envers Dieu saisit Luzina. En venant à la Petite Poule d'Eau où il n'y avait qu' une maison dans laquelle se retirer, Dieu ne s'en était-il pas remis à elle, la seule femme dans l'île!... Et son caur s'était attaché à cette petite fête qui, sans qu'elle le comprît parfaitement, poétisait sa maison comme, autrefois, chez les premiers chrétiens, les agapes fraternelles. (PPE, p. 249)
\end{abstract}

En célébrant la messe dans cet humble décor, le prêtre se met physiquement sur le même plan que les fideles (p. 253). Bien qu'on puisse supposer qu'il disait la messe en latin, sa conviction que tout homme [...] avait le droit d'entendre parler de Dieu dans sa propre langue (p. 261), ce qui l'amène à résumer son sermon en anglais au bénéfice de Tom MacKenzie, nous suggère qu'il n'aurait pas hésité à embrasser les réformes linguistiques promues par le concile.

La critique de l'Église traditionnelle, l'appel d'un renouveau, l'imagination des formes que celui-ci pourrait prendre, tout cet aspect de la Petite Poule d'eau relie ce roman à son successeur et constitue une source peu remarquée de continuité entre les deux ouvrages. Dans Alexandre Chenevert, est approfondi surtout le thème de l'apostolat du laïc. De tous les personnages de Gabrielle Roy, c'est à Chenevert (et après lui à Pierre Cadorai de la Montagne secrète) qu'elle accorde la tâche d'interroger Dieu. C'est lui, tout invraisemblable que cela puisse paraître de prime abord, qu'elle investit du manteau du prophète.

Paul Socken a montré tout ce qui rattache ce petit caissier montréalais, le petit homme de la cage $n^{\circ} 2$ (AC, p. 39), au mythe du héros ${ }^{12}$. Nombreuses sont les allusions qui nous invitent aussi à le rapprocher des prophètes. La comparaison, dès le début, à Gandhi, avec lequel il s'est découvert une ressemblance physique (p. 10), est loin d'être fortuite. La comparaison est reprise vers la fin du roman quand Alexandre songe à jeûner en mémoire du Mahatma qui vient d'être assassiné - un geste qui sera ridiculisé par sa femme, d'esprit plus étroit, moins œcuménique que le sien: On jeûne pour Notre-Seigneur; on ne jeûne pas pour un Hindou (p. 288). Le rappel du pape Pie XII qui non plus ne dormait pas beaucoup (p. 11) est à peine moins significatif. Du prophète, Alexandre possède aussi l'entêtement à penser, à rester lucide, et l'esprit critique, le mérite d' une franchise terrible (p. 149; cf. la redoutable franchise, p. 362), le goût courageux pour la vérité (p. 336). Le docteur Hudon est étonné

12 Paul Socken, «Les dimensions mythiques dans Alexandre Chenevert», Etudes littéraires, vol. XVII, no 3 , hiver 1984, p. 499-529. Texte repris en anglais dans Paul Socken, Myth and Morality in Alexandre Chenevert by Gabrielle Roy, Frankfurt-am-Main, Peter Lang, 1987, p. 11-36. 
de ce dernier trait dans cet homme qu'il avait vu si scandalisé par les mensonges courants (p. 336). Du prophète, il tient aussi par sa dignité (une grande dignité se peignit sur le visage torturé d'Alexandre, p. 336), par l'imagination et la compassion qui lui permettent de s'identifier avec l'humanité, surtout avec l'humanité souffrante.

Avant tout, il partage avec les prophètes l'aptitude qu'il possède - ou plus précisément qu'il acquiert peu à peu au cours de ses derniers mois de vie - à témoigner. Déjà, face à face avec son patron, monsieur Fontaine,

il ne demandait rien. Il n' accusait personne. Cependant, il témoignait. À sa manière, il démontrait que les hommes [...] n'étaient pas tous doués pour le bonheur. [...] Têtu, absorbé et muet, il déposait contre les principes de M. Fontaine, et celui-ci se sentait menacé. (p. 97-98)

Devant le docteur Hudon, c'est une autre sorte de témoignage qu'il porte, celui d'être innombrable, c'est-à-dire de représenter toutes les victimes de l'ennui de la vie moderne dans les grandes villes. Quoique représentatif à cet égard, il se distingue très nettement, néanmoins, par son obstination à penser. Dans leur discussion de ce problème, plus précisément dans les efforts que fait le docteur pour empêcher son client de penser (p. 172), il y a des échos de Pascal - et c'est non pas le médecin mais le malade qui se trouve ici du côté pascalien. Parmi les calmants recommandés par le docteur pour empêcher de penser, se trouvent jetés, plus ou moins dans le même sac, le golf, les quilles et la foi religieuse (p. 170-172).

Témoin au sens le plus profond, le plus «prophétique», Alexandre le devient à la fin, au plus fort de sa douleur physique et au seuil de la mort, la drogue aidant probablement à sa contestation extrêmement audacieuse ${ }^{13}$. Souhaitant sa mort comme un bienfait, est-il dit de ses amis, peut-être souhaitaient-ils aussi que fût soustrait à leurs yeux ce témoignage inquiétant de Dieu (p. 382, c'est moi qui souligne). Phrase mystérieuse, qu'il faut éclairer, je pense, à la lumière de l'extraordinaire affrontement entre le laïc Alexandre et son confesseur, l'abbé Marchand.

Pour arriver à ce point dans l'accomplissement de son rôle de prophète, il fallait un difficile apprentissage. Il fallait que ce petit bourgeois neurasthénique et hypocondriaque, irritable et égocentrique, plein d' amour pour son prochain lorsqu'il le voyait [...] de loin, sans visage, et qui s'éloignait (p. 25) - il fallait qu'il se retire dans le désert. Son désert, paradoxalement, c'est le lac Vert. C'est là qu'il connaît pour la première fois la solitude, la possibilité de bonheur, même de joie, une certitude de Dieu telle que jamais encore il n'en avait éprouvée (p. 215); c'est là qu'il découvre en lui autant de promesses d' inconnu que chez un étranger (p. 207). Grâce la plus précieuse, il reçoit, comme les prophètes, l'appel, la vocation, quoiqu'il ignore exactement de quelle vocation il s'agit. 
La solitude parut absence. [...] Pourtant, au centre de cette absence, il y avait comme un regard qui ne perdait aucun geste, aucune pensée d'Alexandre Chenevert. Était-ce Dieu qui, par cette nuit profonde, au fond des savanes, avait encore repéré Alexandre? Quelle pouvait être la raison d'une attention si tenace? Que voulait Dieu au sujet d'Alexandre qui était en vacances? (p. 204)

Ce que Dieu voulait de lui - mais il n'en avait encore que le plus vague pressentiment - , c'était ce dernier témoignage inquiétant, celui que ne pouvait porter qu'un grand malade. Inquiétant parce qu'il rappelle aux autres ce à quoi ils préferent ne pas penser: leur vulnérabilité, leur mortalité, et surtout, la persistance contre toute apparence, à travers une douleur indéchiffrable (comme aurait dit le père Joseph-Marie), du mystère d'un Dieu qui aime. Fortifié par son expérience au lac Vert, appuyé par la bonté de ses amis (c'est par le détour de l'amour humain qu'il découvre l'amour divin), Alexandre fait face à l'épreuve dernière, rendant son témoignage, dans ses actes comme dans ses paroles, avec cette autorité que peut seule donner la maladie supportée avec dignité et courage. De même que l'œil de Dieu s'était fixé sur Alexandre, à son tour son ceil troubla des inconnus (p. 279).

$\mathrm{Au}$ cœur du dénouement et de la résolution du roman, se trouve l'extraordinaire série de rencontres avec l'abbé Marchand. Ayant formé dans tout ce qui précède l'esprit prophétique de son protagoniste, comment l'auteure le fait-elle servir de signe avant-coureur d'une Église renouvelée? Essentiellement en lui faisant - à lui, laïc - tenir tête à l'autorité cléricale incamée par l'aumônier, pour affirmer sa propre spiritualité, son propre apostolat. Qu'on ne se méprenne pas sur les doutes d'Alexandre touchant la foi catholique. Loin de relever d'un agnosticisme, encore moins d'un athéisme caché, ce sont les doutes d'un croyant, d'autant plus près de Dieu qu'il ose, comme les prophètes, L'interroger. Rejetant les réponses conventionnelles, automatiques que débite l'abbé avec les meilleures intentions, écartant ses objections, Alexandre ose affirmer un humanisme qui finira par lui permettre de se réconcilier avec Dieu.

Mais ce n'est pas seulement par ses paroles qu'Alexandre porte son témoignage inquiétant de Dieu. C'est aussi, et surtout, par ses actes. Mais il fut dérangé dans ses pensées par la voix de l'opéré qui, derrière l'autre paravent, gémissait: "Qu' est-ce que j" ai fait au bon Dieu? » (p. 330) Il se lève, va vers cette solitude, demande à son voisin s'il souffre, prend sa main, la serre, se présente, lui demande s'il a soif, reste avec lui, découvrant tout à coup la fraternité dans la souffrance (p. 330-331). Ce simple geste de compassion relève, bien sûr, de l'humanisme qu'il vient de professer; mais son action dépasse l'humanisme pour rejoindre l'Évangile, comme nous invitent à le croire les nombreux échos bibliques. Mon Dieu, mon Dieu, pourquoi m'as-tu abandonné?; Jésus qui touche les malades ${ }^{14} ; I^{\prime}$ ai soif; $N e$ pouvez-vous pas veiller une heure avec moi?; J'étais malade et vous m'avez visité.

14 Sur le rôle du contact physique dans le roman et l'envie qu'éprouve le docteur Hudon de mettre la main sur l'épaule de M. Chenevert (AC, p. 177), scène qui fait 
Ce qui donne sa beauté à cette très courte scène - l'une des plus puissantes et émouvantes du roman québécois -, c'est à la fois la façon dont elle enchaîne avec les pensées précédentes d'Alexandre (Comment savoir qu'on aime! À quoi le reconnnaître! Est-ce que je vous aime? demanda-t-il à Dieu, p. 330) et l'antithèse qu'elle fait avec la longue scène qui l'a précédée, où sont surtout évidents chez l'abbé le manque de compassion, l'incompréhension. Quels que soient les doutes qui tourmentent Alexandre, le conduisant même au bord du désespoir, c'est lui qui est le vrai prêtre ici. L'abbé Marchand - détail qui vient confirmer cet apostolat - finira par rendre les armes en avouant, devant le problème du mal qui hante l'esprit de son pénitent: Je ne sais pas ce que cela signifie. L'agonie d'Alexandre avait enlevé d̀ sa foi toute prétention orgueilleuse. (p. 380) II est, en d'autres termes, le premier "converti» d'Alexandre - et peut-être pas le dernier.

Le prophète est celui qui parle avant les autres, qui parle au nom de Dieu, en transmettant un message divin ou surnaturel15. Il est évident que Gabrielle Roy n'est pas ni n'a jamais prétendu être prophète dans ce sens-là, une nouvelle Miriam ou Hanna. C'est l'analogie qui nous invite à la rapprocher, elle, l'artisteprophète, des prophètes bibliques. Elle parle au nom d'une vocation qui la pousse parfois malgré elle vers la création littéraire, se disant être la proie de quelque profonde exigence intérieure (DE, p. 211). Je reçois une commande, a-t-elle dit à Donald Cameron ${ }^{16}$. Elle ne reçoit pas la parole de Dieu directement, mais la source où elle puise son inspiration est également mystérieuse, car elle relève en grande partie de l'inconscient, de cette pénombre dont elle a parlé dans une interview avec Gérard Bessette: Je me fie d cette force d la fois obscure et clairvoyante. ${ }^{17} \mathrm{Ne}$ pourrait-on pas sous ce rapport la rapprocher de nouveau de son porte-parole prophétique Chenevert (abstraction faite de la drogue, bien sûr), de ce Chenevert mourant, dont la parole, comme le dit Vachon, émerge d' un niveau de conscience beaucoup plus profond que la conscience claire? ${ }^{18}$

Comme les prophètes aussi, cette artiste - ancienne institutrice, faut-il le rappeler - est motivée par le désir de guider un peuple qu'on aime, ce qui veut dire, en fin de compte, l'humanité. Les bons livres sont ceux qu'on écrit pour, a-t-elle confié à son mari vers la fin de sa vie. Faut d'abord être pour quelque chose. Faut aimer. Et si vous n'aimez pas, ça ne sert à rien d'écrire. On n'écrit pas pour soi, on écrit pour les autres. 19

prévoir l' influence qu'Alexandre exercera sur les autres à la fin du roman, voir Paul Socken, loc. cit., p. 506.

15 Pour la description du prophète biblique, j'ai consulté l'article «Prophet, Prophecy» dans John. L. McKenzie, Dictionary of the Bible, New York et Londres, Macmillan, 1965, ainsi que l'article «Prophetism», de Carl Rahner, dir., Encyclopedia of Theology, New York, Crossroad, 1982.

16 Donald Cameron, Conversations With Canadian Novelists, vol. 2, Toronto Macmillan, 1973, p. 132.

17 Gérard Bessette, Une littérature en ébullition, Montréal, Éditions du Jour, 1967. p. 306. À propos de la «pénombre», voir p. 308.

18 Georges-André Vachon, loc. ćit., p. 273.

19 Entrevue avec Marcel Carbotte, le Devoir, 6 octobre 1984, p. 28. 
Prophetic critic, écrit la théologienne catholique Rosemary Ruether, must be based on constant discernment of the realities of the usigns of the time». We must ask about what is actually happening, not just discuss theoretical constructs divorced from reality. ${ }^{20}$ Qui mieux que certains romanciers sait reconnaître ces «signes du temps»? Si Gabrielle Roy, en particulier, a si bien anticipé le renouveau du catholicisme inspiré par le concile, c'est grâce surtout à sa vision de romancière. Mais c'est aussi parce que la crise traversée par l'Église elle-même à la veille du concile correspond à son évolution personnelle comme catholique. Dans la Détresse et l'enchantement, elle se rappelle ainsi l'Église de sa jeunesse: On eût dit parfois que nous vivions dans quelque enceinte des guerres religieuses, quelque Albi assiégée ou autre cité malheureuse protégée de tous côtés par des défenses, des barbacanes, des interdits. (p. 139) (On dirait l'image que l'Église a eue d'elle-même, en examinant sa conscience, en se regardant dans le miroir du concile.)

Racontant la mort de sa sœur Anna, elle écrit: Je n'étais pas encore tout à fait revenue à la foi de ma jeunesse dont $m$ ' avait éloignée, d̀ ce que je croyais, une église autoritaire, injuste et bornée. (DE, p. 164) Elle poursuit ce thème dans le récit de la messe à laquelle elle a assisté pour plaire à sa mère, la veille de son départ pour l'Europe: Je m'étais peu à peu éloignée de la pratique religieuse, en révolte, à la fin, contre un esprit qui voyait le mal partout, réclamait pour lui seul la possession de la vérité et nous eût tenus à l'écart, s'il l'avait pu, de tout échange avec la généreuse disparité humaine. (DE, p. 238) Combien elle aurait voulu, comme sa mère, avoir assez de foi pour ne pas s'attarder aux errances toutes humaines de l'Eglise, gardant les yeux fixés sur son centre lumineux (DE, p. 238). C'est précisément ce centre lumineux, ce noyau de lumière (DE, p. 240), que le concile s'est donné pour tâche de libérer, en reprenant le chemin de l'Évangile. Et c'est pour ne pas avoir perdu de vue ellemême ce centre lumineux que Gabrielle Roy a pu entrevoir, voire solliciter, à travers les «agapes» de Luzina et la «Passion» d'Alexandre Chenevert, le renouveau à venir.

20 Rosemay Ruether, Contemporary Roman Catholicism: Crises and Challenges, Kansas City, Sheed \& Ward, 1987, p. 60. 